\title{
EMULSION POLYMERIZATION OF THIOPHENE - THE NEW WAY OF CONDUCTING POLYMERS SYNTHESIS
}

\author{
Jacek Nowaczyk ${ }^{1}$, Kornelia Kadac' ${ }^{1}$ Ewa Olewnik-Kruszkowska' ${ }^{1}$ \\ 1 Faculty of Chemistry, Nicolaus Copernicus University in Toruń, Gagarina 7, 78-100 Toruń, Poland, e-mail: \\ jacek.nowaczyk@chem.umk.pl; k_kadac@doktorant.umk.pl; ewa.olewnik@umk.pl
}

Received: 2015.06 .25

Accepted: 2015.08.05

Published: 2015.09.01

\begin{abstract}
The aim of this study was to investigate the new method of polythiophene polymerization. It was made an attempt of the chemical oxidation of thiophene in aqueous medium without the addition of any surfactant. The oxidative compound applied in the synthesis was gaseous ozone. Resulting polymer was obtained in the form of nanospheres dispersed in water. The method developed in this study allow to obtain the polythiophene nanoparticles. Further study is required to fully identify the resulting product properties in comparison with polymer obtained in other methods.
\end{abstract}

Keywords: polythiophene, emulsion polymerization, ozone.

\section{INTRODUCTION}

Polythiophene and its derivatives are among the most important intrinsically conducting polymers. Polythiophene (PTh) has been widely studied since the first reports of their synthesis in scientific literature $[1,2]$. Both environmental and thermal stability of this class of polymers granted in high potential application in different technical processes and products. These polymers have found to be useful in manufacturing of electrooptical devices [3], anticorrosive layers [4], electrochemical sensors and electroactive membranes. Unsubstituted PTh is a hard to process material, due to strong intermolecular interactions, it cannot be melted or dissolved in common solvents. The solution for such drawback was found in incorporating variety of substituents on the polythiophene backbone such as alkyl, alkoxyl, perfluoroalkyl, amine, and carbonyl. Soluble derivatives of PTh can be easily used in different devices via spin-coating or dip-coating. However, recently some effort have been made to synthesize unsubstituted PTh in a form allowing its processability.

In general, three approaches to polymerization of PTh can be distinguished, i.e.: 1) electropolymerization, 2) metal catalyzed coupling reac- tions, and 3) chemical oxidative polymerization. In the case of unsubstituted PTh the first method, reported initially by Diaz et. al. [5], is most often utilized for in-situ polymerization of controlled thickness PTh layers in microelectronic devices. The second method is commonly used in polymerization of substituted polythiophene derivatives with highly regular chain structure, while the last method is used to quickly and easily synthetize PTh and its derivatives having disordered chain structure. Initially this method was reported by Sugimoto [6] and the oxidizing agent was $\mathrm{FeCl}_{3}$. The reaction was carried over in organic solvents most often in dichloromethane. At the beginning of XXI century Fall et. al. [7] have undertaken an attempt to prepare PTh derivatives by chemical oxidative polymerization in aqueous media. First successful attempt of similar synthesis the group have reported in 1998 year and it concerned electropolymerization of poly(3-metylthiophene) in aqueous micellar media [8]. However chemical oxidative polymerization of thiophene and its derivatives in aqueous medium has not been extensively till the presence.

The synthesis of conducting polymer colloid is an attractive both from economic and environmental point of view. First of all, due to rejection 
of expensive and toxic organic solvent and utilization of water. Moreover, the end product is in a form of water based colloid, which can easily be transformed into a respective paint. This way the processability difficulties of polythiophene vanishes. The only weakness of this method is the presence of surfactants which are impossible to get rid of.

Typically, in chemical oxidative polymerization as oxidizing agents iron(III) copper(II), hydroxylperoxide and peroxidisulfate ions are used. The colloid is established and stabilized using adequate surfactant-stabilizer couple.

In this work we make an attempt to synthesize PTh in water based colloid without surfactants and stabilizers. The only factor that keeps the monomer dispersed in water is intensive physically mixed continuous phase. As the oxidizing agent ozone was used, however, the main oxidizing agents were active products of water oxidation. Ozone has one of the highest oxidation potentials i.e.: $2.07 \mathrm{~V}$. Depending on the $\mathrm{pH}$, it reacts with water according to following half-reactions:

$$
\begin{aligned}
& \mathrm{O}_{3}+2 \mathrm{H}^{+}+2 \mathrm{e}^{-} \rightarrow \mathrm{O}_{2}+\mathrm{H}_{2} \mathrm{O} \\
& \mathrm{O}_{3}+\mathrm{H}_{2} \mathrm{O}+\mathrm{e}^{-} \rightarrow \mathrm{O}_{2}+2 \mathrm{OH}^{-}
\end{aligned}
$$

the presence of elevated concentration of hydroxyl anion triggers the reaction of ozone decomposition which yield active species such as hydrogen peroxide and free radicals, especially hydroxyl radicals. The reaction of ozone with hydroxyl anion

$$
\mathrm{O}_{3}+\mathrm{OH}^{-} \rightarrow \mathrm{HO}_{2}^{\bullet}+2 \mathrm{O}^{\bullet}
$$

is considered to be the initiation reaction in the ozone decomposition chain reaction in pure water postulated by Staehelin, Hoigné, and Bühler [9]. Since the lifespan of the radicals is extremely short and reactivity very high they are assumed to be the initiators of the polymerization. However, the question arises if these radicals can survive in water long enough to diffuse through the water monomer interface and react in organic interior of micelle.

Ultimately, the approach appears to be strongly directed toward "green chemistry", both due to water as the synthesis medium and ozone as the main reagent.

Recently similar syntheses were proposed for polyaniline and polypyrrole [10-13]. The second advantage of this method is a product in form of nano-spheres, that remains dispersed in colloidal phase. In this form polymer can be easily used to form the paint or printer ink.
The main objective of the work was to synthesize polythiophene nanoparticles dispersed in water without the help of any surfactants and using ozone as the initial oxidizing reagent.

\section{MATERIALS AND METHODS}

Thiophene monomer was obtained from Sigma Aldrich and used as provided. Deionized double distilled water was used as the solvent. Ozone was obtained by the flowing pure dried oxygen through Corona discharge ozone generator ELTO giving output ozone concentration of about $3 \times 10^{-3}$ $\mathrm{mol} \cdot \mathrm{dm}^{-3} \mathrm{O}_{3}$ in $\mathrm{O}_{2}$. Microscope images were recorded using the scanning electron microscope/ focused ion beam Quanta 3D FEG.

The monomer (thiophene, $35 \mathrm{mmol}$ ) was introduced slowly to quickfit glass reactor of 250 $\mathrm{ml}$ total volume, containing $170 \mathrm{ml}$ of vigorously stirred water. White emulsion of monomer in water has formed instantly. Then, a $\mathrm{pH}$-sensor, and thermometer were introduced to the reactor and ozone flow has been started. Oxygen was provided to the ozone generator at constant flow rate of 2 liters/minute. The oxygen/ozone mixture was bubbled through the reactor for $30 \mathrm{~min}$. After that the remaining ozone was removed by gently blowing a stream of air for $2 \mathrm{~min}$. The reaction was performed in room temperature and the changes of temperature and $\mathrm{pH}$ in reaction mixture was recorded. After the reaction resulting mixture was studied under microscope.

\section{RESULTS AND DISCUSSION}

Monomer was dispersed in water to form small micelles stabilized by continuous stirring of the mixture. Ozone bubbled through the mixture was partially dissolved in water and reacting with the solvent decomposed following the mechanism derived by Staehelin, Hoigné, and Bühler [9].

Taking in to account the rate of ozone decomposition in water it is likely that the ozone molecules diffuse through the water monomer interface and initiate the polymerization of thiophene according to scheme:

$$
\begin{gathered}
\mathrm{O}_{3}+\mathrm{OH}^{-} \rightarrow \mathrm{HO}_{2}^{\bullet}+2 \mathrm{O}^{\bullet-} \\
\mathrm{Th}+\mathrm{HO}_{2}^{\bullet} \rightarrow \mathrm{Th}^{\bullet+}+\mathrm{O}^{\bullet}+\mathrm{OH}^{-}
\end{gathered}
$$

Th denotes thiophene ring which is the monomer in this process. The reaction (4) mentioned earlier was recalled to give the insight in to the 
process complexity. Products of the reaction (5) are thiophene radical cation, atomic oxygen and hydroxyl anion which cannot be solvated in organic phase and escapes to aqueous continuous phase, where can react with fresh ozone molecule according to reaction (4). Thiophene radical cations fuse together giving rise to the chain growth reaction. In the first act the dimer is formed.

$$
\mathrm{Th}^{\bullet+}+\mathrm{Th}^{\bullet+} \rightarrow(\mathrm{Th})_{2}+2 \mathrm{H}^{+}
$$

Later dimers, having oxidation potential lower than the monomer undergoes further oxidations yielding dimer radical cations. Further conjugation of radical cations produce respective oligomers of thiophene.

$$
\begin{aligned}
& (\mathrm{Th})_{2}+\mathrm{O}_{3} \rightarrow(\mathrm{Th})_{2}{ }^{++}+\mathrm{O}^{\bullet}+\mathrm{OH}^{-} \\
& (\mathrm{Th})_{2}{ }^{++}+(\mathrm{Th})_{2}^{++} \rightarrow(\mathrm{Th})_{4} \cdot+2 \mathrm{H}^{+}
\end{aligned}
$$

The alternative mechanism assume that ozone molecules diffuse into micelle and react with monomer via electrophile addition to thiophene double bonds. The exact mechanism is yet to be recognized, however, oxidizing agent resulting from dissolution of ozone in continuous medium is capable to cross the micelle interface and initiate the oxidative polymerization.

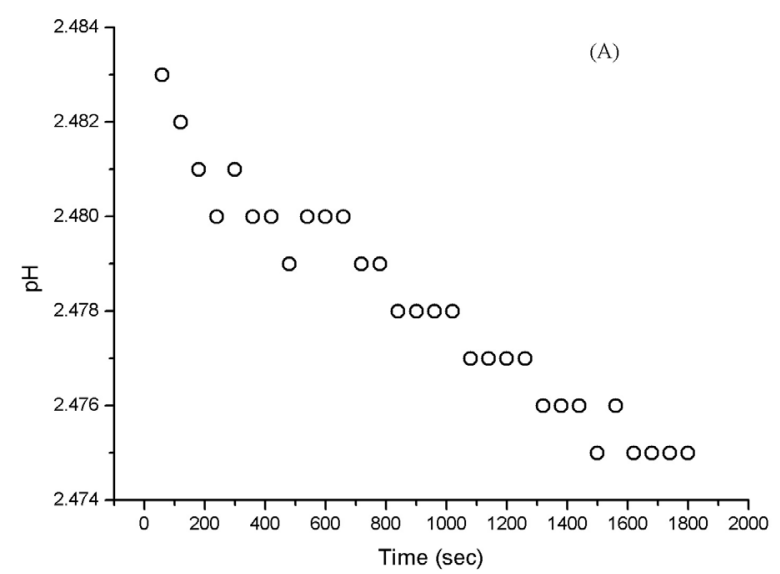

Fig. 1. Recoded changes of $\mathrm{pH}(\mathrm{A})$ and temperature
During the ozone bubbling both the temperature and $\mathrm{pH}$ of the mixture was monitored (see Fig. 1). During the course of the reaction $\mathrm{pH}$ was gradually decreasing and the temperature was rising.

As it is seen from equation (8) binding of two thiophene rings results in a release of two protons. Consequently, the $\mathrm{pH}$ of the mixture decrease, which is observed in the investigated mixture. Calculating the changes in the hydrogen cation concentration it is possible to obtain the relation shown in the Figure 2. The kinetic analysis of the relation is not straightforward since the reaction is a multistage complex process. And the experimental data support this hypothesis because the changes of hydrogen cation concentration does not follow the characteristic kinetic relationships known for the kinetics of first order or second order reactions.

In this experiment ozone was bubbled through the reaction mixture for $30 \mathrm{~min}$. As a result the process milky-like monomer in water emulsion turned to transparent brownish liquid. In the Figure 3 the optical micrographs in polarized light are shown, revealing that in the aqueous continuous phase are dispersed particles of solids.

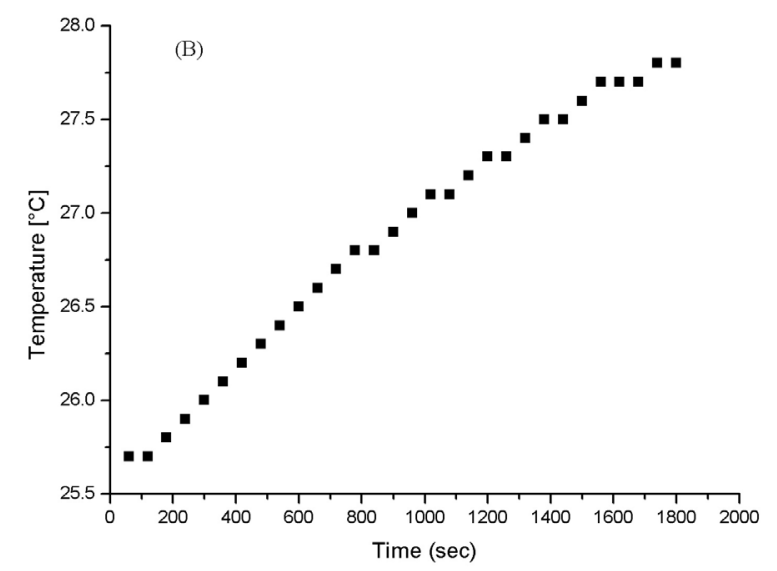

(B) during ozone induced polymerization of thiophene

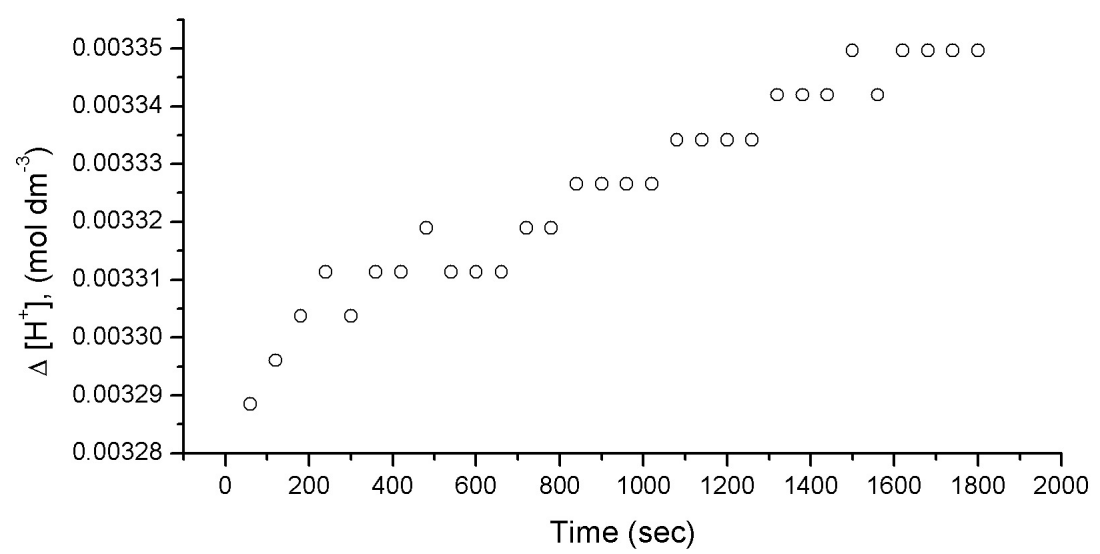

Fig. 2. The change of $\mathrm{H}^{+}$concentration in the reaction mixture during polymerization 


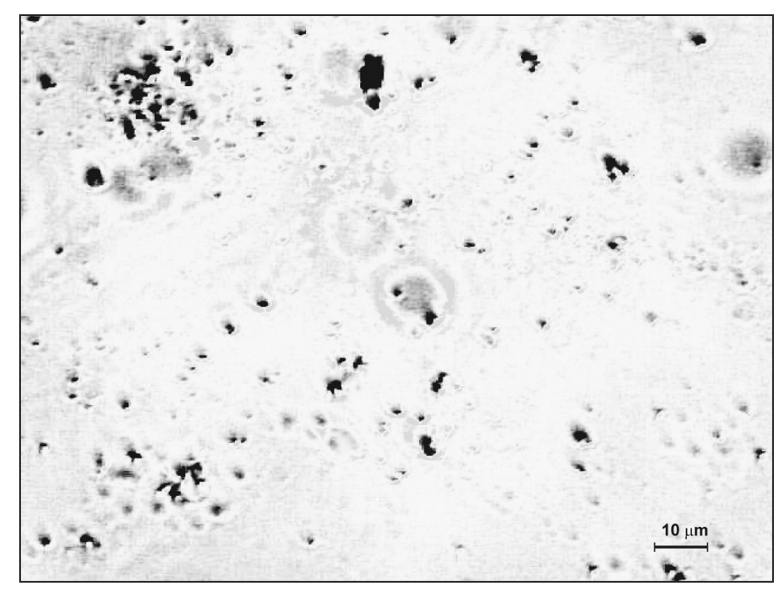

Fig. 3. The microscopy image in polarized light of the liquid phase from reactor after $30 \mathrm{~min}$ of ozone bubbling

The size of polymer particles recorded on the microscopy image ranges from 2 to $8 \mu \mathrm{m}$ with significant excess of small particles having diameter below $5 \mu \mathrm{m}$. After synthesis the mixture was transferred to separatory funnel, and left. The polymers did not settle after one week, what suggests high stability of the resulting dispersion. In the next step the mixture was saturated with $\mathrm{NaCl}$. Salting out did not help to precipitate the polymer. However, this allowed to prepare a specimen for electron microscopy. A droplet of the mixture was placed on a glass plate and evaporated carefully yielding fine microcrystals of $\mathrm{NaCl}$ with enclosed polymer particles (see Fig. 4).

The SEM image presented in Fig. 4. reveals that particles observed in polarized light dispersed in water are actually agglomerated assemblage of small spheroidal grains of polymer of nanometric size.

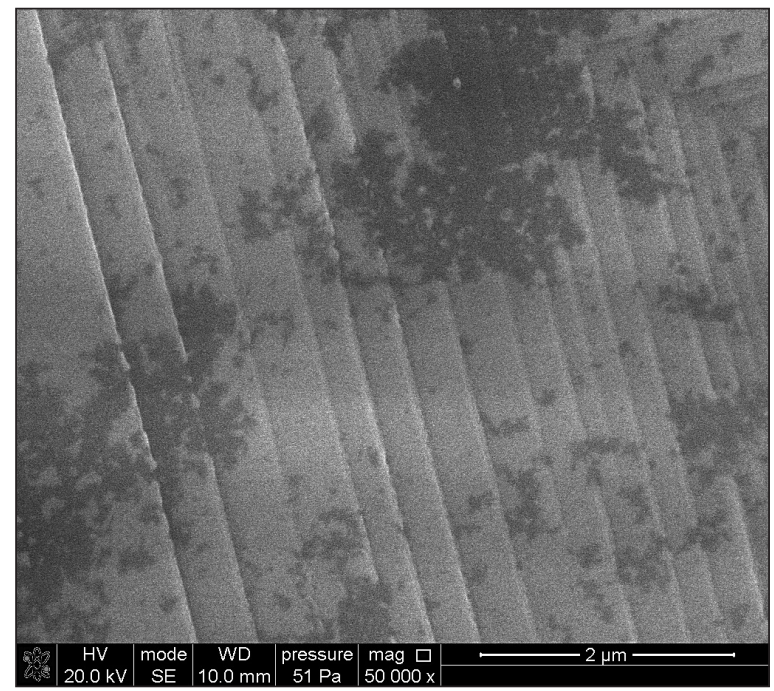

Fig. 4. SEM image of $\mathrm{NaCl}$ crystal with embed polythiophene granules

\section{CONCLUSIONS}

In the present work it was shown that it is possible to obtain polythiophene in the direct chemical oxidation of thiophene by ozone. The reaction conducted in aqueous dispersion results in a stable colloid mixture. The results are promising, however, it is important to undertake further study to optimize the process in the way that allows separation of the polymer and spectroscopic analysis, to find out whether the polymer is not ozone degraded. Also the investigation of synthesis kinetics is interesting from theoretical point of view. The results of the study have shown the new way of the polythiophene synthesis which appears to be interesting and attractive from economic and ecologic point of view.

\section{REFERENCES}

1. Yamamoto T., Sanechika K., Yamamoto A.: Preparation of thermostable and electric-conducting poly(2,5-thienylene). J. Polym. Sci. B Polym. Lett. Ed., 18, 1980, 9-12.

2. Lin J.W.-P., Dudek L.P.: Synthesis and properties of poly(2,5-thienylene). J. Polym. Sci. Polym. Chem. Ed., 18, 1980, 2869-2873.

3. Skotheim T.A., Reynolds J. (Eds): Conjugated polymers: processing and applications. Handbook of Conducting Polymers. Boca Raton, FL, CRC Press 2007.

4. Ocampo C., Armelin E., Liesa F., Alemán C., Ramis X., Iribarren J.I.: Application of a polythiophene derivative as anticorrosive additive for paints. Progress in Organic Coatings, 53, 3, 2005, 217-224.

5. Diaz A.F., Crowley J., Bargon J., Gardini G.P., Torrance J. B.: Electrooxidation of aromatic oligomers and conducting polymers. Journal of Electroanalytical Chemistry, 121, 1981, 355-361.

6. Sugimoto R., Takeda S., et al.: Preparation of soluble polythiophene derivatives utilizing transition metal halides as catalysts and their property. Chemistry Express, 1, 11, 1986, 635-638.

7. Fall M., Diagne A.A., Dieng M.M., Deflorian F., Rossi S., Bonora P.L., Della Volpe C., Aaron J.-J.: Electrochemical impedance spectroscopy of poly(3methoxythiophene) thin films in aqueous $\mathrm{LiClO}_{4}$ solutions. Synthetic Metals, 155, 3, 2005, 569-575.

8. Fall M., Aaron J.J., Sakmeche N., Dieng M.M., Jouini M., Aeiyach S., Lacroix J.C., Lacaze P.C.: Electrochemical and spectroscopic properties of poly(3-methoxythiophene) electrosynthesized in an aqueous micellar medium. Synthetic Metals, 93, 3, 1997, 175-179, 
9. Buehler R. E., Staehelin J., Hoigne J.: Ozone decomposition in water studied by pulse radiolysis. 1. Perhydroxyl $\left(\mathrm{HO}_{2}\right)$ /hyperoxide $\left(\mathrm{O}_{2}^{-}\right)$and $\mathrm{HO}_{3} /$ $\mathrm{O}_{3}{ }^{-}$as intermediates. J. Phys. Chem., 88, 12, 1984, 2560-2564.

10. Wang Y., Chen K., Li T., Li H., Zeng R., Zhang R., Gu Y., Ding J., Liu H.: Soluble polyaniline nanofibers prepared via surfactant-free emulsion polymerization. Synthetic Metals, 198, 2014, 293-299.
11. Kane M., Krafcik K.: Nanostructured soluble conducting polyaniline produced by emulsion polymerization. Synthetic Metals, 181, 2013, 129-135.

12. Oriakhi C.O., Lerner M.M.: Poly(pyrrole) and poly(thiophene)/claynanocomposites via latex-colloid interaction. Materials Research Bulletin 30, 6, 1995, 723-729.

13. Suryawanshi A.J., et al.: Advances in Science and Technology, 79, 2012, 7. 